\title{
nTMS guidance of awake surgery for highly eloquent gliomas
}

\author{
Sebastian Ille, MD, Jens Gempt, MD, Bernhard Meyer, MD, and Sandro M. Krieg, MD, MBA
}

Department of Neurosurgery, Klinikum rechts der Isar, Technische Universität München, Munich, Germany

Navigated transcranial magnetic stimulation (nTMS) allows for preoperative mapping for eloquent gliomas. Besides surgical planning, it also guides intraoperative stimulation mapping. The authors' routine includes preoperative nTMS plus nTMS-based tractography for motor and language to consult patients, plan surgery, craniotomy, and guide cortical and subcortical stimulation.

Here, the authors present this routine in a 48-year-old woman with a glioma of the left middle and superior frontal gyrus reaching the precentral gyrus and superior longitudinal fascicle. Gross-total resection via awake surgery was achieved without deficit.

The nTMS data and nTMS-based tractography augment eloquent glioma management far beyond its current application.

The video can be found here: https://youtu.be/h4ldgMXL1ys.

KEYWORDS awake surgery; DCS; language mapping; motor mapping; nTMS; video 